\title{
Boundary Control of an Axially Moving Steel Strip under a Spatiotemporally Varying Tension*
}

\author{
Kyung-Jinn YANG ${ }^{* *}$, Keum-Shik HONG*** and Fumitoshi MATSUNO**
}

\begin{abstract}
In this paper, a vibration suppression scheme of an axially moving steel strip in the zinc galvanizing line is investigated. The moving steel strip is modeled as a moving beam, in which the tension applied to the strip is a spatiotemporally varying function. The transverse vibration of the strip is controlled by a hydraulic touch-roll actuator at the right boundary. The mathematical model of the system, which consists of a hyperbolic partial differential equation describing the dynamics of the moving beam and an ordinary differential equation describing the actuator dynamics, is derived by using the Hamilton's principle for the systems of changing mass. The Lyapunov method is employed to design a boundary control law for ensuring the vibration reduction of the system in the presence of a spatiotemporally varying tension. The exponential stability of the closed loop system under the boundary control is proved through the use of invariance principle and semigroup theory. Simulation results verify the effectiveness of the boundary control law proposed.
\end{abstract}

Key Words: Axially Moving System, Boundary Control, Hyperbolic Partial Differential Equation, Stability, Periodic System

\section{Introduction}

Figure 1 shows the continuous hot-dip zinc galvanizing process. The steel strips, of order of $1-1.2 \mathrm{~m}$ wide by $0.8-3.0 \mathrm{~mm}$ thick, are preheated and passed at a constant speed through a pot of molten zinc at a temperature in the region of about $450^{\circ} \mathrm{C}$. A zinc film is entrained onto the strip as it emerges from the pot. In order to achieve the target deposited mass and maintain it over various process conditions, a pair of air knives, which direct a long thin wedge-shaped jet of high-velocity air onto the strip, are generally used to control the thickness of the deposited zinc by stripping out excess zinc back into the pot. The deposited film solidifies while the strip moves vertically upward, cooling as it goes, and horizontally for about $110 \mathrm{~m}$, to the gauge that measures the mass of zinc deposited on the strip surfaces.

* Received 9th December, 2003 (No. 03-4194)

** Department of Mechanical Engineering and Intelligent Systems, University of Electro-Communications, 1-5-1 Chofugaoka, Chofu, Tokyo 182-8585, Japan. E-mail: jinnky@hi.mce.uec.ac.jp, matsuno@hi.mce.uec. ac.jp

*** School of Mechanical Engineering, Pusan National University, 30 Changjeon-dong Kumjeong-ku, Busan, 609735, Korea. E-mail: kshong@pusan.ac.kr
Two control objectives in the galvanizing line are to improve the uniformity of the zinc deposit on the strip surfaces and to reduce the zinc consumption. The transverse movement or vibrations of the strip is known to be the main cause for the difference between the average deposited masses on the right and left surfaces and the nonuniformity across the surfaces. As a regulation problem of deposited mass, a number of adjustment methods of the gap between the strip and air knives have been studied by several researchers: Bertin et al. ${ }^{(1)}$, McKerrow ${ }^{(2)}$, Jacobs

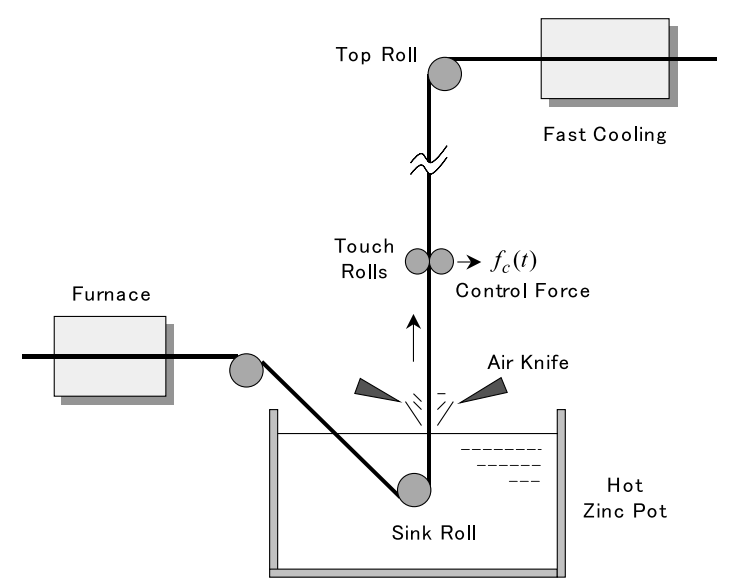

Fig. 1 An axially moving steel strip in the zinc galvanizing line 
and $\mathrm{Chen}^{(3)}$, and $\mathrm{Chen}^{(4)}$. However, a pertinacious problem was the lack of a precise knowledge on strip position due to the vibrations of the strip. Many galvanized steel manufacturers including POSCO (Korea) and U.S. Steel have attempted to measure the strip position directly by installing laser transducers near the air knives. However, no long-term success has been reported yet, because the transducers were unreliable in the high-temperature environment in the long run.

Thus, as an alternative method not using unreliable transducers, the strip vibrations need to be directly suppressed by using a more practical and reasonable method such as active boundary force control. The mathematical models describing the dynamics of a moving steel strip with a hydraulic touch-roll actuator for exerting boundary control force are represented as a coupled hyperbolic partial differential equation (PDE) and an ordinary differential equation (ODE), respectively. The coupled system of a PDE and an ODE can then be analyzed through the use of Lyapunov method and semigroup theory to ensure the stability of the closed loop system and to derive a boundary control law for reducing the vibration energy of the strip.

Axially moving systems can be found in various engineering areas: high-speed magnetic tapes, band saws, power transmission chains and belts, and paper sheets under processing. Especially, the dynamics analysis and control for axially moving beams have received a growing attention due to the entrance of new applications in flexible robotic manipulators and flexible space structures ${ }^{(5)-(9)}$. Particularly, vibration control schemes on axially moving strings include Yang et al. ${ }^{(10)}$, Chung and $\operatorname{Tan}^{(11)}$, Lee and Mote ${ }^{(12)}$, Fung et al. ${ }^{(13)}$, and Li et al. ${ }^{(14)}$, and those on axially moving beams include Choi et al. ${ }^{(15)}$, Hong et al. ${ }^{(16)}$, Lee and Mote ${ }^{(17)}, \mathrm{Li}$ and Rahn ${ }^{(18)}$, and Fung et al. ${ }^{(19)}$

The boundary force control has several advantages over control schemes acting within the spatial domain (e.g., distributed force control). A boundary control law, as apposed to a distributed control law, is not only easily implementable by active or semi-active means at the boundary, but also the dynamic model of the system equation is not altered by adding sensors and actuators. Lee and Mote ${ }^{(17)}$ analyzed a boundary controller to minimize the vibration energy of a translating tensioned beam via an active or passive damping. $\mathrm{Li}$ and $\mathrm{Rahn}^{(18)}$ reported an innovative boundary control strategy to suppress vibrations of an axially moving beam by distinguishing the controlled span from a disturbed span via an active, pivoting roller actuator. Fung et al. ${ }^{(19)}$ developed an optimal boundary control strategy for an axially moving material system through a mass-spring-damper controller. In all papers above, the control laws have been designed under the assumption of a constant tension. However, in practical situations, almost every axially moving system may have an axial tension which is a function of both time and space due to the eccentricity of a support roller, and/or external disturbances, and/or gravity etc. ${ }^{(20)}$ Thus, to obtain a better performance, a boundary controller in the presence of a spatiotemporally varying tension has to be investigated.

The contributions of this paper are: The zinc galvanizing line is analyzed and a control-oriented beam model for the traveling steel strip is derived. Considering the length of the strip, the longitudinal tension of the strip is treated as a spatiotemporally varying function, which is assumed to be bounded and periodic in time. An active vibration suppression scheme in the form of a boundary control and the Lyapunov method are proposed to control the traveling strip system under a spatiotemporally varying tension using a hydraulic touch-roll actuator at the right boundary. The asymptotic and furthermore exponential stability of the closed loop system are assured through the invariance principle and semigroup theory, respectively.

The structure of this paper is organized as follows. In section 2, the governing equation and boundary conditions of an axially moving steel strip are derived by using Hamilton's principle. The boundary control problem is then formulated. In sections 3, a robust boundary force control law is proposed by using the Lyapunov method. In section 4, the asymptotic and exponential stability of the closed loop system are investigated. In section 5, computer simulations are provided. Conclusions are given in section 6.

\section{Problem Formulation: Equations of Motion}

Figure 2 shows a schematic of the axially moving steel strip for control system design purpose. The left boundary at the sink roll is assumed fixed, i.e., fixed in the sense that there is no vertical movement but it allows the strip to move in the horizontal direction. The two touch rolls located in the middle section, but rather close to the sink roll, of the strip are considered to be the right boundary, where the control input (force) is exerted with a hydraulic actuator. In the zinc galvanizing line, the moving steel strip can be modeled as a moving beam with high flexibility.

Let $t$ be the time, $x$ be the spatial coordinate along the longitude of motion, $v_{s}$ be the axial speed of the strip, $w(x, t)$ be the transversal displacement of the strip at spa-

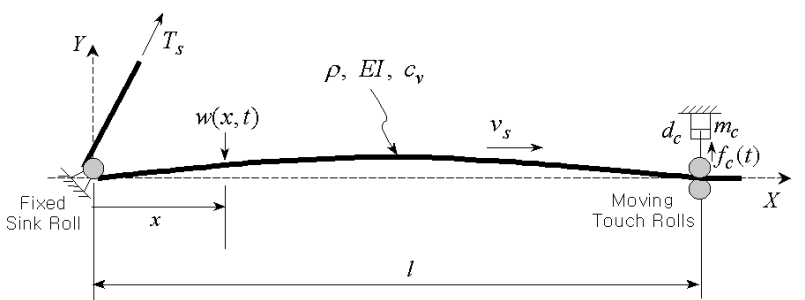

Fig. 2 A control-oriented schematic of the axially moving steel strip with a hydraulic actuator at the right boundary 
tial coordinate $x$ and time $t$, and $l$ be the length of the strip from left to right boundaries. Also, let $\rho$ be the mass per unit length, $T_{s}(x, t)$ be the tension applied to the strip, $E I$ be the flexural rigidity of the strip, and $c_{v}$ be the viscous damping coefficient of the strip. Let the mass and damping coefficient of the hydraulic actuator be $m_{c}$ and $d_{c}$, respectively. The control force $f_{c}(t)$ is applied to the touch rolls to suppress the transverse vibrations of the strip.

Because the strip travels with a constant speed $v_{s}$, the total derivative operator (the material derivative) with respect to time should be defined as $(\cdot) \triangleq d(\cdot) / d t=(\cdot)_{t}+$ $v_{s}(\cdot)_{x}^{(21),(22)}$, where $(\cdot)_{t}=\partial(\cdot) / \partial x$ and $(\cdot)_{x}=\partial(\cdot) / \partial x$ denote the partial derivatives.

The kinetic energy of the steel strip between $x=0$ and $x=l$ including the hydraulic actuator is

$$
T=\frac{1}{2} \int_{0}^{l} \rho\left\{v_{s}^{2}+\left(v_{s} w_{x}+w_{t}\right)^{2}\right\} d x+\frac{1}{2} m_{c} w_{t}^{2}(l, t) .
$$

For brevity, $w_{x}=w_{x}(x, t)$ and $w_{t}=w_{t}(x, t)$ have been used, and similar abbreviations will be used in the sequel. The potential energy of the system is

$$
V=\frac{1}{2} \int_{0}^{l} T_{s}(x, t) w_{x}^{2} d x+\frac{1}{2} \int_{0}^{l} E I w_{x x}^{2} d x,
$$

where $w_{x x}=w_{x x}(x, t)$. The virtual work by the external forces is

$$
\begin{gathered}
\delta W=f_{c} \delta w(l, t)-\int_{0}^{l} c_{v}\left(w_{t}+v_{s} w_{x}\right) \delta w d x \\
-d_{c} w_{t}(l, t) \delta w(l, t) .
\end{gathered}
$$

Now, by using the Hamilton's principle, i.e., $\int_{t_{0}}^{t_{1}}(\delta T-$ $\delta V+\delta W) d t=0$, the governing equation and boundary conditions are derived as follows:

$$
\begin{aligned}
& \rho w_{t t}(x, t)+2 \rho v_{s} w_{x t}(x, t)+\rho v_{s}^{2} w_{x x}(x, t) \\
& \quad-\left(T_{s}(x, t) w_{x}(x, t)\right)_{x}+c_{v}\left(w_{t}(x, t)+v_{s} w_{x}(x, t)\right) \\
& \quad+E I w_{x x x x}(x, t)=0, \quad 0<x<l, \\
& w(x, 0)=w_{0}(x), \quad w_{t}(x, 0)=w_{t 0}(x), \\
& w(0, t)=w_{x}(0, t)=0, \quad w_{x}(l, t)=0,
\end{aligned}
$$

and

$$
\begin{aligned}
f_{c} & =m_{c} w_{t t}(l, t)+\left(d_{c}-\rho v_{s}\right) w_{t}(l, t) \\
& +\left(T_{s}(l, t)-\rho v_{s}^{2}\right) w_{x}(l, t)-E I w_{x x x}(l, t) .
\end{aligned}
$$

(4) is a linear hyperbolic PDE governing the transverse motion $w(x, t)$ of the axially moving strip. (5) and (6) - (7) denote the initial conditions and the boundary conditions of the system, respectively. Note that the right boundary condition (7) is an ODE that describes the equation of motion of the hydraulic actuator in compliance with the transversal force at $x=l$. For notational brevity, all variables at boundaries will be written without explicit time in the sequel, i.e., $w(0)=w(0, t), w(l)=w(l, t), T_{s}(l)=T_{s}(l, t)$, etc.
As shown in (2) and (4), the tension $T_{s}(x, t)$ is a spatiotemporally, but periodic in time, varying function. Since the steel strip is moving vertically, the gravitational force $\rho g x$, which acts as an additional tension to the strip, cannot be neglected. Also the tension itself may be timevarying due to the eccentricity of a support roller, which causes a periodic excitation. Thus, these variations need to be incorporated in control system design. It is now assumed that $T_{s}(x, t)$ is sufficiently smooth and uniformly bounded as follows:

$$
\begin{aligned}
& T_{s, \min } \leq T_{s}(x, t) \leq T_{s, \max }, \\
& \left|\left(T_{s}(x, t)\right)_{t}\right| \leq\left(T_{s}\right)_{t, \max }, \\
& \left|\left(T_{s}(x, t)\right)_{x}\right| \leq\left(T_{s}\right)_{x, \max },
\end{aligned}
$$

for all $x \in[0, l], t \geq 0$, and some a priori known constants $T_{s, \min }, T_{s, \max },\left(T_{s}\right)_{t, \max }$, and $\left(T_{s}\right)_{x, \max }$.

The objective of a boundary control is now to stabilize asymptotically the vibration energy of the axially moving strip with a time-varying condition at $x=l$. From (1) $-(2)$, the mechanical energy $E_{m}(t)$ is given by

$$
\begin{gathered}
E_{m}(t)=\frac{1}{2} \int_{0}^{l} \rho\left\{v_{s}^{2}+\left(v_{s} w_{x}+w_{t}\right)^{2}\right\} d x+\frac{1}{2} \int_{0}^{l} T_{s} w_{x}^{2} d x \\
+\frac{1}{2} \int_{0}^{l} E I w_{x x}^{2} d x+\frac{1}{2} m_{c} w_{t}^{2}(l),
\end{gathered}
$$

where the traveling speed of the strip, $v_{s}$, is a constant and $T_{s}=T_{s}(x, t)$.

In the remainder of this section, to order to give a general idea regarding how the boundary control action will work, the dynamics of the moving strip with both fixed boundaries is first analyzed. In this case, the boundary conditions in (6) and (7) become $w(0)=w_{x}(0)=w(l)=$ $w_{x}(l)=0$, and henceforth $m_{c} w_{t}^{2}(l)=0$ in (11). The time derivative of $E_{m}(t)$ in (11) is now evaluated by applying the one-dimensional transport theorem of moving material $^{(23)}$ as follows:

$$
\begin{aligned}
\dot{E}_{m}(t) & =\frac{1}{2} \int_{0}^{l} 2 \rho\left(w_{t}+v_{s} w_{x}\right)\left(w_{t t}+2 v_{s} w_{x t}+v_{s}^{2} w_{x x}\right) d x \\
& +\int_{0}^{l} T_{s} w_{x}\left(w_{x t}+v_{s} w_{x x}\right) d x \\
& +\frac{1}{2} \int_{0}^{l}\left\{\left(T_{s}\right)_{t}+v_{s}\left(T_{s}\right)_{x}\right\} w_{x}^{2} d x \\
& +\int_{0}^{l} E I w_{x x}\left(w_{t}+v_{s} w_{x}\right)_{x x} d x \\
& =\int_{0}^{l}\left(w_{t}+v_{s} w_{x}\right)\left(\left(T_{s} w_{x}\right)_{x}\right. \\
& \left.-c_{v}\left(w_{x}+v_{s} w_{x}\right)-E I w_{x x x x}\right) d x \\
& +\int_{0}^{l} T_{s} w_{x}\left(w_{x t}+v_{s} w_{x x}\right) d x \\
& +\frac{1}{2} \int_{0}^{l}\left\{\left(T_{s}\right)_{t}+v_{s}\left(T_{s}\right)_{x}\right\} w_{x}^{2} d x \\
& +\int_{0}^{l} E I w_{x x}\left(w_{t}+v_{s} w_{x}\right)_{x x} d x
\end{aligned}
$$


where (4) has been used in deriving the second equality. Integrating by parts, the terms in (12) are further simplified as follows:

$$
\begin{aligned}
& \int_{0}^{l}\left\{w_{t}\left(T_{s} w_{x}\right)_{x}+T_{s} w_{x} w_{x t}\right\} d x=\left[w_{t}\left(T_{s} w_{x}\right)\right]_{0}^{l} \\
& \int_{0}^{l} w_{x}\left(T_{s} w_{x}\right)_{x} d x=\int_{0}^{l} \frac{d}{d x}\left(T_{s} w_{x}^{2}\right) d x-\int_{0}^{l} T_{s} w_{x} w_{x x} d x \\
& =\left[T_{s} w_{x}^{2}\right]_{0}^{l}-\int_{0}^{l} T_{s} w_{x} w_{x x} d x \\
& \int_{0}^{l} w_{x x x x}\left(w_{t}+v_{s} w_{x}\right) d x=\left[w_{x x x}\left(w_{t}+v_{s} w_{x}\right)\right]_{0}^{l} \\
& -\left[w_{x x}\left(w_{t}+v_{s} w_{x}\right)_{x}\right]_{0}^{l}+\int_{0}^{l} w_{x x}\left(w_{t}+v_{s} w_{x}\right)_{x x} d x
\end{aligned}
$$

The substitution of (13)-(15), together with the fixed boundary conditions, into (12) yields:

$$
\begin{gathered}
\dot{E}_{m}(t)=-c_{v} \int_{0}^{l}\left(w_{t}+v_{s} w_{x}\right)^{2} d x-v_{s} E I w_{x x}^{2}(0) \\
+v_{s} E I w_{x x}^{2}(l)+\frac{1}{2} \int_{0}^{l}\left\{\left(T_{s}\right)_{t}+v_{s}\left(T_{s}\right)_{x}\right\} w_{x}^{2} d x .
\end{gathered}
$$

Now, the following observations from (16) are made: (i) The viscous damping reduces the mechanical energy of the axially moving strip, which can be seen from the existence of $-c_{v} \int_{0}^{l}\left(w_{t}+v_{s} w_{x}\right)^{2} d x$. (ii) Even though the transverse velocities and the slopes at both boundaries are zero, i.e., $w_{t}(0)=w_{t}(l)=w_{x}(0)=w_{x}(l)=0$, the instantaneous bending moment, $v_{s} E I w_{x x}$, can still deform the strip. The bending moment at $x=0$ decreases the mechanical energy, while that at $x=l$ increases it. (iii) The time derivative of $T_{s}(x, t),\left(T_{s}\right)_{t}+v_{s}\left(T_{s}\right)_{x}$, increases the energy flux by the factor of $w_{x}^{2}$. The last observation indicates that the time rate of the change of $T_{s}(x, t)$ cannot be simply neglected.

Thus, it is concluded that for the traveling strip with fixed boundary conditions, the bending moment occurring at the right boundary $x=l$ and the time rate of the change of tension $T_{s}(x, t)$ should be treated properly to decrease $E_{m}(t)$.

\section{Design of Boundary Control Law}

The selection of a suitable Lyapunov function and the construction of an effective control input are most important issues in the Lyapunov method. To obtain the asymptotic stability of system (4)-(7), the convergence of the hydraulic actuator displacement $w(l)$ to zero should also be satisfied. But the mechanical energy $E_{m}(t)$ in (11) does not involve a state variable of the hydraulic actuator, $w(l)$. Thus, a modification of (11) is pursued.

Note that by boundary conditions (6) and Poincare's inequality $^{(24)}$, there exists a positive constant $C_{1}$ such that

$$
\int_{0}^{l} w_{0}^{2} d x \leq C_{1} \int_{0}^{l} w_{x}^{2} d x \text { for } x \in[0, l] .
$$

From (17), it is seen that the convergence of the transverse displacement $w(x, t)$ for $x \in[0, l]$ to zero can be obtained by considering the convergence of the slope $w_{x}(x, t)$ for $x \in[0, l]$. That is, if $\lim _{t \rightarrow \infty} \int_{0}^{l} w_{x}^{2} d x=0$ is satisfied, then $\lim _{t \rightarrow \infty} \int_{0}^{l} w_{0}^{2} d x=0$ is also satisfied. Thus, the asymptotic stability of the hydraulic actuator system can be analyzed by adding the slope term at $x=l$ in the mechanical energy.

The following positive definite functional $V_{m}(t)$, replacing $E_{m}(t)$ in (11), is now considered.

$$
\begin{gathered}
V_{m}(t)=\frac{1}{2} \int_{0}^{l} \rho\left(v_{s} w_{x}+w_{t}\right)^{2} d x+\frac{1}{2} \int_{0}^{l} T_{s} w_{x}^{2} d x \\
+\frac{1}{2} \int_{0}^{l} E I w_{x x}^{2} d x+\frac{1}{2} m_{c}\left\{w_{t}^{2}(l)+w_{x}^{2}(l)\right\} .
\end{gathered}
$$

Note that the constant term $v_{s}^{2}$ has been omitted in (18) because it doesn't make any difference in deriving the final control law. It is also noted that there exist positive constants $C_{2}$ and $C_{3}$ such that the following holds:

$$
\begin{gathered}
C_{2}\left\{w_{t}^{2}(l)+w_{x}^{2}(l)+\int_{0}^{l} w_{x}^{2} d x\right\} \\
\leq\left\{w_{t}(l)+w_{x}(l)\right\}^{2}+\int_{0}^{l} w_{x}^{2} d x \\
\quad \leq C_{3}\left\{w_{t}^{2}(l)+w_{x}^{2}(l)+\int_{0}^{l} w_{x}^{2} d x\right\} .
\end{gathered}
$$

Using inequality (19), some positive constants $C_{4}$ and $C_{5}$ can be chosen, so that the following holds:

$$
\begin{aligned}
C_{4} & {\left[\frac{1}{2} m_{c}\left\{w_{t}^{2}(l)+w_{x}^{2}(l)\right\}+\frac{1}{2} \int_{0}^{l} T_{s} w_{x}^{2} d x\right] } \\
& \leq \frac{1}{2 \alpha} m_{c}\left\{\alpha w_{t}(l)+\left(\alpha v_{s}+2 \beta l\right) w_{x}(l)\right\}^{2}+\frac{1}{2} \int_{0}^{l} T_{s} w_{x}^{2} d x \\
& \leq C_{5}\left[\frac{1}{2} m_{c}\left\{w_{t}^{2}(l)+w_{x}^{2}(l)\right\}+\frac{1}{2} \int_{0}^{l} T_{s} w_{x}^{2} d x\right]
\end{aligned}
$$

where $\alpha$ and $\beta$ are positive constants.

Now, a further modification of the positive definite functional, $V_{0}(t)$, is proposed as follows:

$$
\begin{aligned}
& V_{0}(t)=\frac{1}{2} \int_{0}^{l} \rho\left(v_{s} w_{x}+w_{t}\right)^{2} d x+\frac{1}{2} \int_{0}^{l} T_{s} w_{x}^{2} d x \\
&+\frac{1}{2} \int_{0}^{l} E I w_{x x}^{2} d x+\frac{1}{2 \alpha} m_{c}\left\{\alpha w_{t}(l)+\left(\alpha v_{s}+2 \beta l\right) w_{x}(l)\right\}^{2} .
\end{aligned}
$$

From (18), (20) and (21), the following holds:

$$
C_{4} V_{m}(t) \leq V_{0}(t) \leq C_{5} V_{m}(t),
$$

which means that $V_{0}(t)$ is equivalent to $V_{m}(t)$.

Using $V_{0}(t)$, define a functional $V(t)$ such that

$$
V(t)=\alpha V_{0}(t)+2 \beta \int_{0}^{l} \rho x w_{x}\left(w_{t}+v_{s} w_{x}\right) d x
$$




$$
\begin{aligned}
= & \alpha V_{0}(t)+\beta \int_{0}^{l} \rho x\left\{w_{x}+\left(w_{t}+v_{s} w_{x}\right)\right\}^{2} d x \\
& -\beta \int_{0}^{l} \rho x w_{x}^{2} d x-\beta \int_{0}^{l} \rho x\left(w_{t}+v_{s} w_{x}\right)^{2} d x,
\end{aligned}
$$

where

$$
\begin{aligned}
& \frac{\alpha}{2} \int_{0}^{l} \rho\left(w_{t}+v_{s} w_{x}\right)^{2} d x \geq \beta \int_{0}^{l} \rho l\left(w_{t}+v_{s} w_{x}\right)^{2} d x \\
& \quad \geq \beta \int_{0}^{l} \rho x\left(w_{t}+v_{s} w_{x}\right)^{2} d x \\
& \frac{\alpha}{2} \int_{0}^{l} T_{s}(x, t) w_{x}^{2} d x \geq \frac{\alpha}{2} T_{s, \min } \int_{0}^{l} w_{x}^{2} d x \geq \beta \int_{0}^{l} \rho l w_{x}^{2} d x \\
& \quad \geq \beta \int_{0}^{l} \rho x w_{x}^{2} d x .
\end{aligned}
$$

From (25) and (26), it is seen that $V(t)$ is a positive definite functional if $\alpha>2 \beta l$ and $T_{s, \min }>\rho$.

By using the Cauchy-Schwarz inequality, it can also be shown that there exists a positive constant $C_{6}$ such that

$$
\begin{aligned}
2 \beta & \int_{0}^{l} \rho x w_{x}\left(v_{s} w_{x}+w_{t}\right) d x \\
& \leq \beta \rho l\left\{\int_{0}^{l} w_{x}^{2} d x+\int_{0}^{l}\left(w_{t}+v_{s} w_{x}\right)^{2} d x\right\} \\
& \leq C_{6}\left\{\frac{1}{2} T_{s, \min } \int_{0}^{l} w_{x}^{2} d x+\frac{1}{2} \int_{0}^{l} \rho\left(w_{t}+v_{s} w_{x}\right)^{2} d x\right\} \\
& \leq C_{6}\left\{\frac{1}{2} \int_{0}^{l} T_{s} w_{x}^{2} d x+\frac{1}{2} \int_{0}^{l} \rho\left(w_{t}+v_{s} w_{x}\right)^{2} d x\right\} \\
& \leq C_{6} V_{0}(t),
\end{aligned}
$$

where $C_{6} \geq \max \left(\frac{2 \beta \rho l}{T_{s, \min }}, 2 \beta l\right)$. From (21), (23) and (27), the following holds:

$$
\left(\alpha-C_{6}\right) V_{0}(t) \leq V(t) \leq\left(\alpha+C_{6}\right) V_{0}(t),
$$

where $\alpha>\max \left(\frac{2 \beta \rho l}{T_{s, \min }}, 2 \beta l\right)$. From (28), it is concluded that $V(t)$ in (23) is equivalent to the positive definite functional $V_{0}(t)$ in $(21)$.

In this paper, the positive definite functional $V(t)$ in (23) is considered as a Lyapunov function candidate for system (4) - (7) to determine a boundary force control law for the stabilization of the vibration energy $E_{m}(t)$ in (11) and to obtain the asymptotic stability of the closed loop system.

The time derivative of $V(t)$ along (4) yields:

$$
\begin{aligned}
& \dot{V}(t)=\alpha \dot{V}_{0}+\frac{d}{d t}\left[2 \beta \int_{0}^{l} \rho x w_{x}\left(w_{t}+v_{s} w_{x}\right) d x\right] \\
& =\alpha \dot{V}_{0}(t)+2 \beta \rho \int_{0}^{l} x\left(w_{x t}+v_{s} w_{x x}\right)\left(w_{t}+v_{s} w_{x}\right) d x \\
& \quad+2 \beta \rho \int_{0}^{l} x w_{x}\left(w_{t t}+2 v_{s} w_{x t}+v_{s}^{2} w_{x x}\right) d x \\
& \quad+2 \beta \rho v_{s} \int_{0}^{l} w_{x}\left(w_{t}+v_{s} w_{x}\right) d x
\end{aligned}
$$

$$
\begin{aligned}
& =\alpha \dot{V}_{0}(t)+\beta \rho\left[x\left(w_{t}+v_{s} w_{x}\right)^{2}\right]_{0}^{l} \\
& -\beta \rho \int_{0}^{l}\left(w_{t}+v_{s} w_{x}\right)^{2} d x \\
& +2 \beta \int_{0}^{l} x w_{x}\left(\left(T_{s} w_{x}\right)_{x}-c_{v}\left(w_{x}+v_{s} w_{x}\right)-E I w_{x x x x}\right) d x \\
& +2 \beta \rho v_{s} \int_{0}^{l} w_{x}\left(w_{t}+v_{s} w_{x}\right) d x
\end{aligned}
$$

The integration by parts yields:

$$
\begin{aligned}
& 2 \int_{0}^{l} x w_{x}\left(T_{s} w_{x}\right)_{x} d x=2 \int_{0}^{l} x \frac{d}{d x}\left(T_{s} w_{x}^{2}\right) d x \\
& -2 \int_{0}^{l} x T_{s} w_{x} w_{x x} d x=\left[x\left(T_{s} w_{x}^{2}\right)\right]_{0}^{l} \\
& -\int_{0}^{l} T_{s} w_{x}^{2} d x+\int_{0}^{l} x\left(T_{s}\right)_{x} w_{x}^{2} d x, \\
& -\int_{0}^{l} x w_{x} w_{x x x x} d x=-\left[x w_{x} w_{x x x}\right]_{0}^{l}+\left[w_{x} w_{x x}\right]_{0}^{l} \\
& -\int_{0}^{l} w_{x x}^{2} d x+\int_{0}^{l} x w_{x x} w_{x x x} d x .
\end{aligned}
$$

Since $\left[x w_{x x}^{2}\right]_{0}^{l}=\int_{0}^{l} w_{x x}^{2} d x+2 \int_{0}^{l} x w_{x x} w_{x x x} d x,(31)$ can be rewritten as

$$
\begin{gathered}
-\int_{0}^{l} x w_{x} w_{x x x x} d x=-\left[x w_{x} w_{x x x}\right]_{0}^{l}+\left[w_{x} w_{x x}\right]_{0}^{l} \\
+\left[x w_{x x}^{2}\right]_{0}^{l}-2 \int_{0}^{l} w_{x x}^{2} d x-\int_{0}^{l} x w_{x x} w_{x x x} d x .
\end{gathered}
$$

From (31) and (32), the following is derived:

$$
\begin{aligned}
& -\int_{0}^{l} x w_{x} w_{x x x x} d x=-2\left[x w_{x} w_{x x x}\right]_{0}^{l} \\
& +2\left[w_{x} w_{x x}\right]_{0}^{l}+\left[x w_{x x}^{2}\right]_{0}^{l}-3 \int_{0}^{l} w_{x x}^{2} d x .
\end{aligned}
$$

The following inequality is also utilized.

$$
u v \leq \gamma u^{2}+\frac{1}{\gamma} v^{2}, \text { for any } \gamma>0 .
$$

Using (34), the followings are derived.

$$
\begin{aligned}
& \int_{0}^{l} x w_{x}\left(w_{t}+v_{s} w_{x}\right) d x \\
& \leq l \gamma_{1} \int_{0}^{l} w_{x}^{2} d x+\frac{l}{\gamma_{1}} \int_{0}^{l}\left(w_{t}+v_{s} w_{x}\right)^{2} d x \\
& \int_{0}^{l} w_{x}\left(w_{t}+v_{s} w_{x}\right) d x \\
& \leq \gamma_{2} \int_{0}^{l} w_{x}^{2} d x+\frac{1}{\gamma_{2}} \int_{0}^{l}\left(w_{t}+v_{s} w_{x}\right)^{2} d x
\end{aligned}
$$

where $\gamma_{i}>0, i=1,2$.

Thus, by substituting (4), (6), (8)-(10), (13)-(15), (30), (33), and (35) - (36) into (29), the time derivative of 
the Lyapunov function candidate $V(t)$, with a right boundary actuator at $x=l$ and the fixed condition at left boundary, becomes:

$$
\begin{aligned}
\dot{V}(t) \leq & -\left(\alpha c_{v}+\beta \rho-\frac{2 \beta c_{v} l}{\gamma_{1}}-\frac{2 \beta \rho v_{s}}{\gamma_{2}}\right) \int_{0}^{l}\left(w_{t}+v_{s} w_{x}\right)^{2} d x \\
& -\left\{\beta T_{s, \min }-\left(\beta l+\frac{\alpha v_{s}}{2}\right)\left(T_{s}\right)_{x, \max }-\frac{\alpha}{2}\left(T_{s}\right)_{t, \max }\right. \\
& \left.-2 \beta\left(c_{v} \gamma_{1} l+\rho v_{s} \gamma_{2}\right)\right\} \int_{0}^{l} w_{x}^{2} d x-3 \beta E I \int_{0}^{l} w_{x x}^{2} d x \\
& +\alpha T_{s}(l) w_{x}(l)\left(w_{t}(l)+v_{s} w_{x}(l)\right)-\alpha v_{s} T_{s}(0) w_{x}^{2}(0) \\
& +\beta l T_{s}(l) w_{x}^{2}(l)-\alpha E I w_{x x x}(l)\left(w_{t}(l)+v_{s} w_{x}(l)\right) \\
& +\alpha E I v_{s} w_{x x x}(0) w_{x}(0)+\alpha E I w_{x x}(l)\left(w_{x t}(l)+v_{s} w_{x x}(l)\right) \\
& -\alpha E I v_{s} w_{x x}^{2}(0)-\alpha E I w_{x x}(0) w_{x t}(0) \\
& -2 \beta l E I w_{x}(l) w_{x x x}(l)+2 \beta E I w_{x}(l) w_{x x}(l) \\
& -2 \beta E I w_{x}(0) w_{x x}(0)+\beta l E I w_{x x}^{2}(l)+\beta \rho l\left(w_{t}(l)+v_{s} w_{x}(l)\right)^{2} \\
& +\frac{d}{d t}\left[\frac{1}{2 \alpha} m_{c}\left\{\alpha w_{t}(l)+\left(\alpha v_{s}+2 \beta l\right) w_{x}(l)\right\}^{2}\right] \\
& =-\left(\alpha c_{v}+\beta \rho-\frac{2 \beta c_{v} l}{\gamma_{1}}-\frac{2 \beta \rho v_{s}}{\gamma_{2}}\right) \int_{0}^{l}\left(w_{t}+v_{s} w_{x}\right)^{2} d x \\
& +\frac{d}{d t}\left[\frac{1}{2 \alpha} m_{c}\left\{\alpha w_{t}(l)+\left(\alpha v_{s}+2 \beta l\right) w_{x}(l)\right\}^{2}\right] \\
& +\left\{\beta T_{s, \min }-\left(\beta l+\frac{\alpha v_{s}}{2}\right)\left(T_{s}\right)_{x, \max }-\frac{\alpha}{2}\left(T_{s}\right)_{t, \max }\right. \\
& -2 \beta\left(c_{v} \gamma_{1} l+\rho v_{s}+\beta l\right) T_{s}(l)+\int_{0}^{l} w_{x}^{2} d x \\
& -3 \beta E I v_{x x x}(l)\left\{\alpha w_{t}(l)+\left(\alpha v_{s}+2 \beta l\right) w_{x}\left(l x-\alpha E I v_{s} w_{x x}^{2}(0)\right.\right. \\
& \\
& \\
& \\
&
\end{aligned}
$$

The time derivative, along (7), of the last term on the right in (37) yields:

$$
\begin{aligned}
\frac{d}{d t}[ & \left.\frac{1}{2 \alpha} m_{c}\left\{\alpha w_{t}(l)+\left(\alpha v_{s}+2 \beta l\right) w_{x}(l)\right\}^{2}\right] \\
& =\frac{1}{\alpha} m_{c}\left\{\alpha w_{t}(l)+\left(\alpha v_{s}+2 \beta l\right) w_{x}(l)\right\}\left\{\alpha w_{t t}(l)\right. \\
& \left.+\left(\alpha v_{s}+2 \beta l\right) w_{x t}(l)\right\} \\
& =\left\{\alpha w_{t}(l)+\left(\alpha v_{s}+2 \beta l\right) w_{x}(l)\right\}\left\{f_{c}+\frac{m_{c}}{\alpha}\left(\alpha v_{s}+2 \beta l\right) w_{x t}(l)\right\} \\
& -\alpha\left(d_{c}-\rho v_{s}\right) w_{t}^{2}(l) \\
& -\left\{\left(\alpha v_{s}+2 \beta l\right)\left(d_{c}-\rho v_{s}\right)+\alpha\left(T_{s}(l)-\rho v_{s}^{2}\right)\right\} w_{t}(l) w_{x}(l) \\
& -\left(\alpha v_{s}+2 \beta l\right)\left(T_{s}(l)-\rho v_{s}^{2}\right) w_{x}^{2}(l) \\
& +\left\{\alpha w_{t}(l)+\left(\alpha v_{s}+2 \beta l\right) w_{x}(l)\right\} E I w_{x x x}(l) .
\end{aligned}
$$

A boundary force control law, which makes the time derivative of $V(t)$ negative definite in the presence of the spatiotemporally varying tension, is then proposed as follows:

$$
f_{c}=\left(d_{c}-2 \rho v_{s}\right) w_{t}(l)-\frac{m_{c}}{\alpha}\left(\alpha v_{s}+2 \beta l\right) w_{x t}(l) .
$$

The substitution of (38) and boundary force control law (39) into (37) yields:

$$
\begin{aligned}
\dot{V}(t) & \leq-\left(\alpha c_{v}+\beta \rho-\frac{2 \beta c_{v} l}{\gamma_{1}}-\frac{2 \beta \rho v_{s}}{\gamma_{2}}\right) \int_{0}^{l}\left(w_{t}+v_{s} w_{x}\right)^{2} d x \\
& -\left\{\beta T_{s, \min }-\left(\beta l+\frac{\alpha v_{s}}{2}\right)\left(T_{s}\right)_{x, \max }-\frac{\alpha}{2}\left(T_{s}\right)_{t, \max }\right. \\
& \left.-2 \beta\left(c_{v} \gamma_{1} l+\rho v_{s} \gamma_{2}\right)\right\} \int_{0}^{l} w_{x}^{2} d x \\
& -3 \beta E I \int_{0}^{l} w_{x x}^{2} d x-\alpha E I v_{s} w_{x x}^{2}(0) \\
& -\rho\left(3 \alpha v_{s}-\beta l\right) w_{t}^{2}(l) \\
& -\left\{\beta l T_{s}(l)-\left(\alpha v_{s}+3 \beta l\right) \rho v_{s}^{2}\right\} w_{x}^{2}(l),
\end{aligned}
$$

where $\left(3 \alpha v_{s}-\beta l\right)>0$ and $\left\{\beta l T_{s}(l)-\left(\alpha v_{s}+3 \beta l\right) \rho v_{s}^{2}\right\}>0$.

If $T_{s, \min }$ is sufficiently large, the positive values $\alpha, \beta$, and $\gamma_{i}, i=1,2$, can be chosen to satisfy

$$
\begin{aligned}
& \left(\alpha c_{v}+\beta \rho-\frac{2 \beta c_{v} l}{\gamma_{1}}-\frac{2 \beta \rho v_{s}}{\gamma_{2}}\right)>0, \\
& \left\{\beta T_{s, \min }-\left(\beta l+\frac{\alpha v_{s}}{2}\right)\left(T_{s}\right)_{x, \max }-\frac{\alpha}{2}\left(T_{s}\right)_{t, \text { max }}\right. \\
& \left.\quad-2 \beta\left(c_{v} \gamma_{1} l+\rho v_{s} \gamma_{2}\right)\right\}>0 .
\end{aligned}
$$

Thus, the following is obtained:

$$
\begin{aligned}
\dot{V}(t) & \leq-c_{0}\left(\int_{0}^{l}\left(w_{t}+v_{s} w_{x}\right)^{2} d x+\int_{0}^{l} w_{x}^{2} d x\right. \\
+ & \left.\int_{0}^{l} w_{x x}^{2} d x+w_{t}^{2}(l)+w_{x}^{2}(l)\right)
\end{aligned}
$$

where

$$
\begin{aligned}
c_{0} & =\min \left\{\left(\alpha c_{v}+\beta \rho-\frac{2 \beta c_{v} l}{\gamma_{1}}-\frac{2 \beta \rho v_{s}}{\gamma_{2}}\right),\right. \\
& 3 \beta E I, \rho\left(3 \alpha v_{s}-\beta l\right), \beta l T_{s}(l)-\left(\alpha v_{s}+3 \beta l\right) \rho v_{s}^{2}, \\
& \beta T_{s, \min }-\left(\beta l+\frac{\alpha v_{s}}{2}\right)\left(T_{s}\right)_{x, \max }-\frac{\alpha}{2}\left(T_{s}\right)_{t, \max } \\
& \left.-2 \beta\left(c_{v} \gamma_{1} l+\rho v_{s} \gamma_{2}\right)\right\} .
\end{aligned}
$$

From (43), it is concluded that the positive definite functional $V(t)$ in (23) is non-increasing and is a Lyapunov function, because $\dot{V}(t)$ is negative definite. Hence, all the signals in the closed loop system are bounded.

In the boundary force control law (39), the velocity $w_{t}(l)$ and slope rate $w_{x t}(l)$ at $x=l$ are involved. The slop rate $w_{x t}(l)$ can be implemented by backward differentiation of the signal from an encoder, which measures the slope $w_{x}(l)^{(16)}$.

\section{Stability Analysis}

In this section, the asymptotic and, furthermore, exponential stability of the axially moving strip under 
boundary control law (39) are proven. The substitution of (39) into (7) yields:

$$
\begin{aligned}
& m_{c} w_{t t}(l)+\rho v_{s} w_{t}(l)+\left(T_{s}(l)-\rho v_{s}^{2}\right) w_{x}(l) \\
& \quad-E I w_{x x x}(l)+m_{c} \alpha^{-1}\left(\alpha v_{s}+2 \beta l\right) w_{x t}(l)=0 .
\end{aligned}
$$

In other to analyze the asymptotic stability of the closed loop system (4) - (6) and (44), the state space $\mathfrak{I}$ is defined as follows:

$\mathfrak{J}^{\triangleq}\left\{\left(w, \dot{w}, w(l), w_{t}(l)\right)^{T} \mid w \in H_{L}^{2}, \dot{w} \in L^{2}\right.$, and $\left.w(l), w_{t}(l) \in R\right\}$,

where the superscript $T$ stands for transpose. The spaces $L_{2}$ and $H_{L}^{k}$ are defined as follows:

$$
\begin{aligned}
& L^{2} \triangleq\left\{f:[0, l] \rightarrow R \mid \int_{0}^{l} f^{2} d x<\infty\right\}, \\
& H_{L}^{k} \stackrel{\Delta}{\triangleq}\left\{f \in L^{2} \mid f^{\prime}, f^{\prime \prime}, \cdots, f^{(k)} \in L^{2},\right. \\
& \text { and } f(0)=0\} \text {. }
\end{aligned}
$$

In the space $\mathfrak{I}$, the inner-product is defined as follows:

$$
\begin{aligned}
& \langle z, \hat{z}\rangle_{\mathfrak{T}}=\frac{\Delta}{2} \int_{0}^{l}\left(T_{s} w_{x} \hat{w}_{x}+E I w_{x x} \hat{w}_{x x}\right) d x+\frac{\alpha}{2} \int_{0}^{l} \rho \dot{w} \dot{\hat{w}} d x \\
& \quad+\beta \int_{0}^{l} \rho x\left(w_{x}+\dot{w}\right)\left(\hat{w}_{x}+\dot{\hat{w}}\right) d x \\
& \quad-\beta \int_{0}^{l} \rho x w_{x} \hat{w}_{x} d x-\beta \int_{0}^{l} \rho x \dot{w} \dot{\hat{w}} d x \\
& \quad+\frac{1}{2} m_{c}\left\{\left(\alpha v_{s}+2 \beta l\right) w_{x}(l)+\alpha w_{t}(l)\right\} \\
& \quad \times\left\{\left(\alpha v_{s}+2 \beta l\right) \hat{w}_{x}(l)+\alpha \hat{w}_{t}(l)\right\}, \\
& \quad=\frac{\alpha}{2} \int_{0}^{l}\left(T_{s} w_{x} \hat{w}_{x}+E I w_{x x} \hat{w}_{x x}\right) d x+\frac{\alpha}{2} \int_{0}^{l} \rho \dot{w} \dot{\hat{w}} d x
\end{aligned}
$$

$$
\begin{aligned}
& +\beta \int_{0}^{l} \rho x w_{x} \dot{\hat{w}} d x+\beta \int_{0}^{l} \rho x \dot{w} \hat{w}_{x} d x \\
& +\frac{1}{2} m_{c}\left\{\left(\alpha v_{s}+2 \beta l\right) w_{x}(l)+\alpha w_{t}(l)\right\}\left\{\left(\alpha v_{s}\right.\right. \\
& \left.+2 \beta l) \hat{w}_{x}(l)+\alpha \hat{w}_{t}(l)\right\},
\end{aligned}
$$

where $z=\left(w, \dot{w}, w(l), w_{t}(l)\right)^{T}, \hat{z}=\left(\hat{w}, \dot{\hat{w}}, \hat{w}(l), \hat{w}_{t}(l)\right)^{T} \in \mathfrak{I}$, and $\alpha, \beta>0$. The norm induced by inner-product (48) is equivalent to $V(t)$ in (23), i.e.,

$$
\begin{aligned}
& V(t)=\langle z, z\rangle_{\mathfrak{I}}=\|z(t)\|_{\mathfrak{I}}^{2} \\
& \quad=\frac{\alpha}{2} \int_{0}^{l} \rho \dot{w}^{2} d x+\frac{\alpha}{2} \int_{0}^{l} T_{s} w_{x}^{2} d x+\frac{\alpha}{2} \int_{0}^{l} E I w_{x x}^{2} d x \\
& +2 \beta \int_{0}^{l} \rho x w_{x} \dot{w} d x+\frac{1}{2} m_{c}\left\{\alpha w_{t}(l)+\left(\alpha v_{s}+2 \beta l\right) w_{x}(l)\right\}^{2} .
\end{aligned}
$$

By using

$$
\ddot{w}=\frac{d^{2}}{d t^{2}} w=\frac{d}{d t}\left(w_{t}+v_{s} w_{x}\right)=w_{t t}+2 v_{s} w_{x t}+v_{s}^{2} w_{x x},
$$

the closed loop system (4)-(6) and (44) can be rewritten in the following abstract form.

$$
\dot{z}=A(t) z, \quad z(0) \in \mathfrak{J},
$$

where $z=\left(w, \dot{w}, w(l), w_{t}(l)\right)^{T} \in \mathfrak{I}$. Let

$D \stackrel{\Delta}{=}\left\{\left(w, \dot{w}, w(l), w_{t}(l)\right)^{T} \mid w \in H_{L}^{4}, \dot{w} \in H_{L}^{2}\right.$, and $\left.w(l), w_{t}(l) \in R\right\}$,

and define the family of operators $\{A(t)\}_{t \geq 0}, A: D \subset \mathfrak{I} \rightarrow \mathfrak{J}$ by

$$
A(t) \triangleq\left[\begin{array}{cccc}
\rho^{-1}\left\{\frac{\partial}{\partial x}\left(T_{s} \frac{\partial}{\partial x}\right)-E I \frac{\partial^{4}}{\partial x^{4}}\right\} & -\rho^{-1} c_{v} & 0 & 0 \\
0 & 0 & 0 & 1 \\
-m_{c}^{-1}\left\{\left(T_{s}-\rho v_{s}^{2}\right) \frac{\partial}{\partial x}-E I \frac{\partial^{3}}{\partial x^{3}}\right\}_{x=l} & -\left.\frac{1}{\alpha}\left(\alpha v_{s}+2 \beta l\right) \frac{\partial}{\partial x}\right|_{x=l} & 0 & -m_{c}^{-1} \rho v_{s}
\end{array}\right] .
$$

Since the time derivative of $V(t)$ is negative definite as shown in (43), $A(t)$ given by (51) is dissipative in the space $\mathfrak{I}$ because of $\dot{V}(t)=\langle\dot{z}, z\rangle_{\mathfrak{I}}=\langle A(t) z, z\rangle_{\mathfrak{I}} \leq 0$ for $V(t)=\|z(t)\|_{\mathfrak{I}}^{2}$ using (49) and $\dot{z}=A(t) z$. This implies that $\{A(t)\}_{t \geq 0}$ is a stable family of infinitesimal generators of $C_{0}$ semigroups on $\mathfrak{J}$. Further, the map $t \rightarrow A(t) z$ for $z \in D$ is strongly continuously differentiable in $\mathfrak{I}$. Thus, it follows from Theorem 4.8 in Pazy ${ }^{(25)}$ that there exists a unique evolution system, $\{S(t, s): 0 \leq s \leq t\}$, on $\mathfrak{I}$ associated with the homogeneous system corresponding to (50). That is, the system (50) admits a unique solution $z(t)$ such that

$$
z(t)=S(t, 0) z_{0}, \quad t \geq 0 .
$$

\subsection{Asymptotic stability}

The closed loop system (50) is shown to be asymptotically sable by applying the invariance principle ${ }^{(26)}$ in the state space $\mathfrak{T}$.

Theorem 1. Consider the system (50) representing (4) - (6) and (44). Suppose that the following hold:

$$
\alpha>\max \left(\frac{2 \beta \rho l}{T_{s, \min }}, 2 \beta l\right), \quad T_{s, \min }>\rho, \quad\left(3 \alpha v_{s}-\beta l\right)>0,
$$




$$
\begin{aligned}
& \left\{\beta l T_{s}(l)-\left(\alpha v_{s}+3 \beta l\right) \rho v_{s}^{2}\right\}>0, \\
& \left(\alpha c_{v}+\beta \rho-\frac{2 \beta c_{v} l}{\gamma_{1}}-\frac{2 \beta \rho v_{s}}{\gamma_{2}}\right)>0, \text { and } \\
& \left\{\beta T_{s, \min }-\left(\beta l+\frac{\alpha v_{s}}{2}\right)\left(T_{s}\right)_{x, \max }-\frac{\alpha}{2}\left(T_{s}\right)_{t, \max }\right. \\
& \left.-2 \beta\left(c_{v} \gamma_{1} l+\rho v_{s} \gamma_{2}\right)\right\}>0 .
\end{aligned}
$$

Then, the solution $z(t)$ of system (50) is asymptotically stable, i.e., $z(t) \rightarrow 0$ as $t \rightarrow \infty$, by boundary force control law (39).

Proof. $\quad V(t)$ in (49) is a Lyapunov function on $\mathfrak{J}$ because $\dot{V}(t) \leq 0$ for all $z \in \mathfrak{J}$ as shown in (43). Since the tension $T_{s}(x, t)$ is assumed to be spatiotemporally varying but periodic in time, the system (50) is a periodic system. Thus, a set $B=\{z \in \mathfrak{I} \mid \dot{V}(t)=0\}$ with $B_{m}$, where $B_{m}$ is the maximal invariant subset of $B$, can be defined ${ }^{(26)}$. Then $z(t)=S(t, 0) z_{0} \rightarrow B_{m}$ as $t \rightarrow \infty$ for all $z_{0} \in \mathfrak{J}$, i.e., any solution of system (50) asymptotically approaches to the maximal invariant set $B_{m}$. Any element of $B$, except zero solution, cannot be invariant, because waves of a nonzero solution propagate to $x=l$ with the speed $v_{s}$ by the characteristics of wave equation. Therefore, the maximal invariant set includes only the origin, $B_{m}=\{0\}$, and $z(t)$ is asymptotically stabilized by boundary control law (39) at $x=l$.

\subsection{Exponential stability}

To verify that right boundary force control law (39) induces a rate of exponential decay of $V(t)$, the following theorem is proved using a semigroup of closed-loop system (50).

Theorem 2. Consider system (50) as Theorem 1. Suppose that there exist

$$
\begin{aligned}
& \alpha>\max \left(\frac{2 \beta \rho l}{T_{s, \min }}, 2 \beta l\right), \quad T_{s, \min }>\rho, \quad\left(3 \alpha v_{s}-\beta l\right)>0, \\
&\left\{\beta l T_{s}(l)-\left(\alpha v_{s}+3 \beta l\right) \rho v_{s}^{2}\right\}>0, \\
&\left(\alpha c_{v}+\beta \rho-\frac{2 \beta c_{v} l}{\gamma_{1}}-\frac{2 \beta \rho v_{s}}{\gamma_{2}}\right)>0, \text { and } \\
&\left\{\beta T_{s, \min }-\left(\beta l+\frac{\alpha v_{s}}{2}\right)\left(T_{s}\right)_{x, \max }\right. \\
&\left.-\frac{\alpha}{2}\left(T_{s}\right)_{t, \max }-2 \beta\left(c_{v} \gamma_{1} l+\rho v_{s} \gamma_{2}\right)\right\}>0 .
\end{aligned}
$$

Then, the Lyapunov function $V(t)$ given by (23) decays exponentially to zero along the solution of system (50). That is, there exist constants $\mu>0$ and $M>0$ such that

$$
V(t) \leq M e^{-\mu t}, \quad t \geq 0 .
$$

Proof. There exists a constant $\sigma>0$ such that

$$
\begin{gathered}
\int_{0}^{l}\left(w_{t}+v_{s} w_{x}\right)^{2} d x+\int_{0}^{l} w_{x}^{2} d x \\
+\int_{0}^{l} w_{x x}^{2} d x+w_{t}^{2}(l)+w_{x}^{2}(l)
\end{gathered}
$$

$$
\begin{aligned}
& \geq \sigma\left[\frac{\alpha}{2} \int_{0}^{l} \rho\left(v_{s} w_{x}+w_{t}\right)^{2} d x+\frac{\alpha}{2} \int_{0}^{l} T_{s} w_{x}^{2} d x\right. \\
& \left.+\frac{\alpha}{2} \int_{0}^{l} E I w_{x x}^{2} d x+\frac{1}{2} m_{c}\left\{\alpha w_{t}(l)+\left(\alpha v_{s}+2 \beta l\right) w_{x}(l)\right\}^{2}\right] \\
& =\sigma \alpha V_{0}(t) .
\end{aligned}
$$

From (24) and (54), (43) can be rewritten as

$$
\begin{aligned}
\dot{V}(t) & \leq-c_{0} \sigma \alpha V_{0}(t) \\
& \leq-c_{0} \sigma\left[\alpha V_{0}(t)-\beta \int_{0}^{l} \rho x w_{x}^{2} d x\right. \\
& -\beta \int_{0}^{l} \rho x\left(w_{t}+v_{s} w_{x}\right)^{2} d x \\
& \left.+\beta \int_{0}^{l} \rho x\left\{w_{x}+\left(w_{t}+v_{s} w_{x}\right)\right\}^{2} d x\right] \\
& +c_{0} \sigma \beta \int_{0}^{l} \rho x\left\{w_{x}+\left(w_{t}+v_{s} w_{x}\right)\right\}^{2} d x \\
& \leq-c_{0} \sigma V(t)+c_{0} \sigma \beta \int_{0}^{l} \rho x\left\{w_{x}+\left(w_{t}+v_{s} w_{x}\right)\right\}^{2} d x .
\end{aligned}
$$

Note that from Theorem 1, the closed loop system (50) is asymptotically stable, i.e., $\lim _{t \rightarrow \infty} \int_{0}^{l}\left\{w_{x}+\left(w_{t}+v_{s} w_{x}\right)\right\}^{2} d x=$ 0 , and then $\int_{0}^{\infty} \int_{0}^{l} x\left\{w_{x}+\left(w_{t}+v_{s} w_{x}\right)\right\}^{2} d x d t<\infty$.

Thus, from (49) and (53), the integration of both sides of (55) from 0 to $\infty$ yields:

$$
\begin{aligned}
& \int_{0}^{\infty} V(t) d t=\int_{0}^{\infty}\|z(t)\|_{\mathfrak{J}}^{2} d t=\int_{0}^{\infty}\left\|S(t, 0) z_{0}\right\|_{\mathfrak{J}}^{2} d t \\
& \quad \leq \frac{1}{c_{0} \sigma}(V(0)-V(\infty)) \\
& \quad+\beta \int_{0}^{\infty} \int_{0}^{l} \rho x\left\{w_{x}+\left(w_{t}+v w_{x}\right)\right\}^{2} d x d t<\infty .
\end{aligned}
$$

By (56) and Theorem 4.1 in Pazy ${ }^{(25)}$, there exist $M_{1} \geq 1$ and $\mu_{1}>0$ such that

$$
\|S(t, 0)\|_{\mathfrak{J}} \leq M_{1} e^{-\mu_{1} t} .
$$

The application of (57) to (53) gives

$$
\|z(t)\|_{\mathfrak{I}} \leq M_{1}\left\|z_{0}\right\|_{\mathfrak{I}} e^{-\mu_{1} t}
$$

proving the exponential stability of the solution of system (50). Therefore, the Lyapunov function $V(t)$ also decays exponentially such that

$$
V(t)=\|z(t)\|_{\mathfrak{J}}^{2} \leq M_{1}^{2}\left\|z_{0}\right\|_{\mathfrak{J}}^{2} e^{-2 \mu_{1} t} \leq M e^{-\mu t},
$$

where $M=M_{1}^{2}\left\|z_{0}\right\|_{\mathfrak{J}}^{2}$ and $\mu=2 \mu_{1}$.

\section{Numerical Simulations}

The effectiveness of the proposed boundary control law is illustrated by numerical simulations using a finite difference method. An implicit difference operator is used because the fourth order term $w_{x x x x}(x, t)$ requires a small time increment in an explicit method ${ }^{(17)}$. 


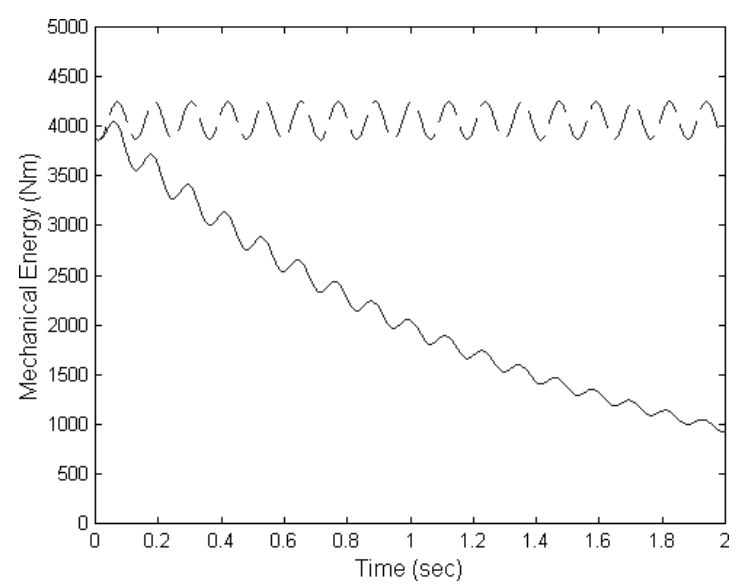

(a) During 1 to 2 seconds

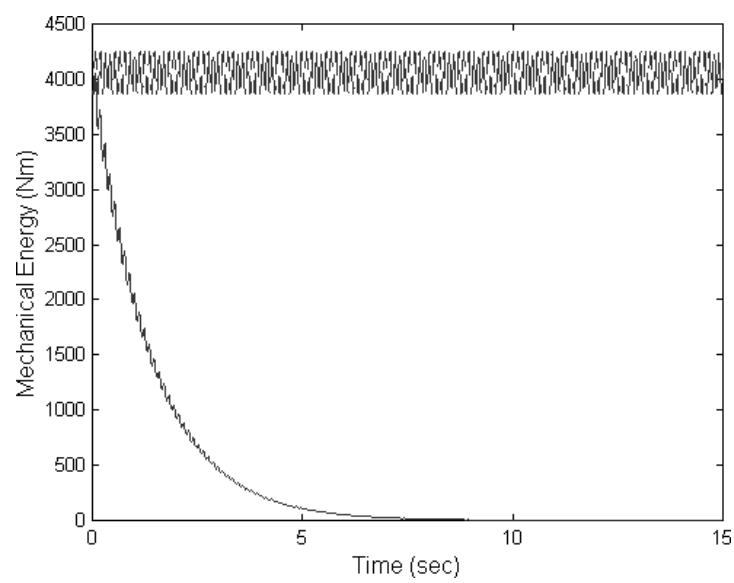

(b) During 1 to 15 seconds

Fig. 3 Comparison of the decays of mechanical energy (11) of the system with boundary force controller (39) (solid line) and the system with no control (dashed line): $\alpha=1$, $\beta=0.01$

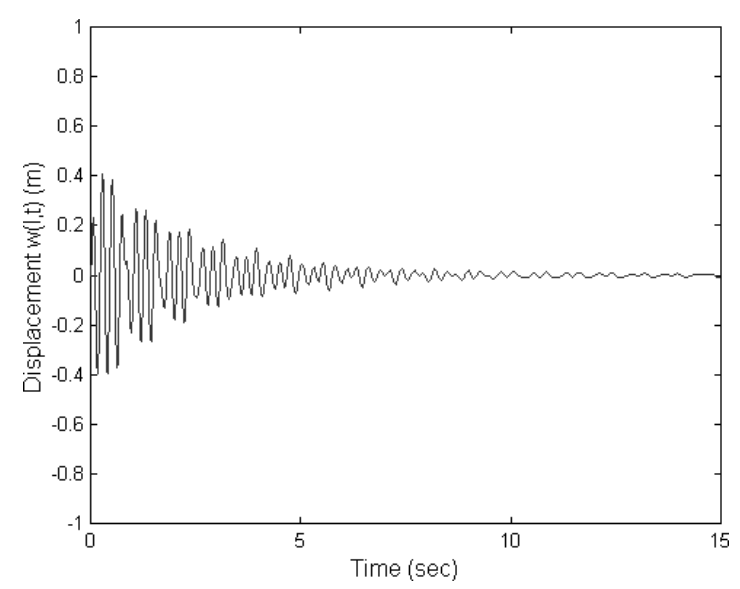

Fig. 4 The transverse displacement at the right boundary, $w(l, t)$

The parameters of the axially moving beam used for numerical simulations are: $\rho=2.7 \mathrm{~kg} / \mathrm{m}, E I=146 \mathrm{Nm}^{2}$, $c_{v}=0.001 \mathrm{Nm}^{2} \mathrm{sec}, l=35 \mathrm{~m}, v_{s}=1 \mathrm{~m} / \mathrm{sec}, m_{c}=10 \mathrm{~kg}, d_{c}=$ $0.25 \mathrm{~N} / \mathrm{m} / \mathrm{sec}$, and $T_{s}=26000+9.8 \rho x+\sin t \mathrm{~N}$. The initial conditions are $w_{0}=0.5 \sin 3 \pi x$ and $w_{t 0}=0$.

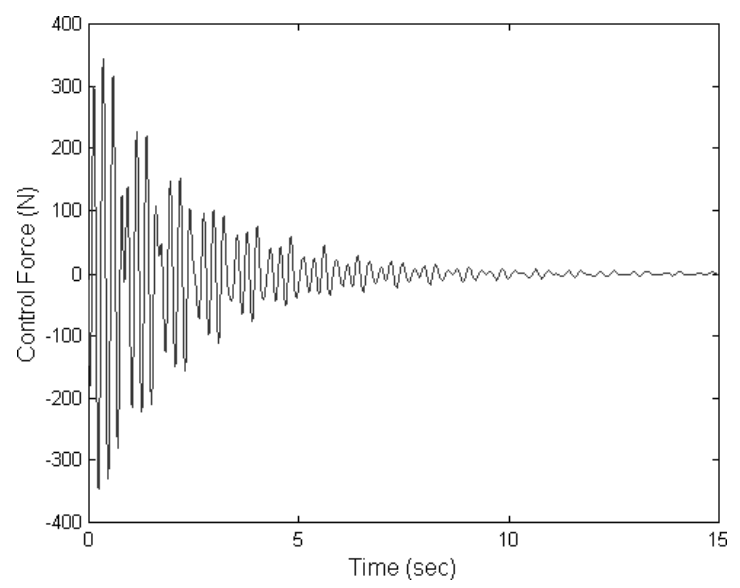

Fig. 5 The control input force used: $f_{c}(t)$

The controller parameters are selected as $\alpha=1, \beta=$ 0.01 , and $\gamma_{1}=\gamma_{2}=10$, from which the conditions in Theorem 1 and Theorem 2 are satisfied, i.e.,

$$
\begin{aligned}
& \alpha>\max \left(\frac{2 \beta \rho l}{T_{s, \min }}, 2 \beta l\right)=0.7, \\
& \left(\alpha c_{v}+\beta \rho-\frac{2 \beta c_{v} l}{\gamma_{1}}-\frac{2 \beta \rho v_{s}}{\gamma_{2}}\right)=0.0225>0, \\
& T_{s, \min }>\rho,\left(3 \alpha v_{s}-\beta l\right)=2.65>0, \\
& \left\{\beta l T_{s}(l)-\left(\alpha v_{s}+3 \beta l\right) \rho v_{s}^{2}\right\}>9400>0, \text { and } \\
& \left\{\beta T_{s, \min }-\left(\beta l+\frac{\alpha v_{s}}{2}\right)\left(T_{s}\right)_{x, \max }\right. \\
& \left.-\frac{\alpha}{2}\left(T_{s}\right)_{t, \max }-2 \beta\left(c_{v} \gamma_{1} l+\rho v_{s} \gamma_{2}\right)\right\}=236.4>0,
\end{aligned}
$$

where $T_{s, \text { min }}=25999 \mathrm{~N},\left(T_{s}\right)_{x, \text { max }}=9.8 \rho \mathrm{N}$, and $\left(T_{s}\right)_{t, \text { max }}=$ $1 \mathrm{~N}$.

A comparison of the mechanical energies, $E_{m}(t)$ of (11), with boundary force control and without any control action is shown in Fig. 3. As analyzed in section 4, the initial vibration energy dissipates exponentially with the control action. However, the strip vibrations without any control remain almost at the same level. Figure 4 shows the displacement at the right boundary, $w(l, t)$. Figure 5 depicts the control force used in getting Fig. 4.

\section{Conclusions}

In this paper, a boundary control scheme to suppress the transverse vibration of an axially moving steel strip with a spatiotemporally varying tension in the hot-dip galvanizing line has been investigated. The following findings are concluded:

( i ) In the case of a traveling strip, even if the displacements, velocities, and slopes at both boundaries are zero, the bending moment occurring at the right boundary, $x=l$, and the time rate of the change of tension $T_{s}(x, t)$ can cause an increase of the total mechanical energy.

(ii ) A boundary force control can achieve the suppression of the vibration of the entire strip in the presence of a spatiotemporally varying tension. The exponen- 
tial stability of the closed loop system with the boundary control law proposed has been proved with the Lyapunov method and semi-group theory.

(iii) Since the feedback terms in the boundary control law are the velocity and slope rate at the right boundary, the vibration suppression of the axially moving strip can be successfully implemented by the boundary control law.

\section{Acknowledgements}

This work was supported by the Ministry of Science and Technology of Korea under the program of National Research Laboratory, grant number NRL M1-0302-000039-03-J00-00-023-10. The first author also would like to thank the Korea Science and Engineering Foundation for the support of his postdoctoral fellowship program in Japan.

\section{References}

( 1 ) Bertin, M.C., Brown, L., Whitehead, R. L. and Yrisarri, E., Computer Control of Coating Weight on Galvanizing and Tinning Lines, Proc. 7th IFAC World Congress, Helsinki, (1978), pp.199-206.

( 2 ) McKerrow, P.J., Computer Controlled Galvanizing, Comput. Industry, Vol.4 (1983), pp.19-30.

( 3 ) Jacobs, O.L.R. and Chen, D., Regulation of Deposited Zinc in Hot-Dip Galvanizing, Proc. European Control Conf., Grenoble, Paper, No.32, (1991).

( 4 ) Chen, D., Adaptive Control of Hot-Dip Galvanizing, Automatica, Vol.31, No.5 (1995), pp.715-733.

( 5 ) Yang, K.J., Hong, K.S., Yoo, W.S. and Matsuno, F., Model Reference Adaptive Control of a Cantilevered Flexible Beam, JSME Int. J., Ser. C, Vol.46, No.2 (2003), pp.640-651.

( 6 ) Yang, K.J., Hong, K.S., Rhee, E.J. and Yoo, W.S., Model Reference Adaptive Control of a Flexible Structure, KSME International Journal, Vol.15, No.10 (2001), pp.1356-1368.

( 7 ) Yang, K.J. and Hong, K.S., Robust MARC of a Nonautonomous Parabolic System with Spatially-Varying Coefficients and Bounded Disturbance, Asian Journal of Control, Vol.5, No.3 (2003), pp.350-363.

( 8 ) Matsuno, F., Ohno, T. and Orlov, Y., Proportional Derivative and Strain (PDS) Boundary Feedback Control of a Flexible Space Structure with a Closed-Loop Chain Mechanism, Automatica, Vol.38, No.7 (2002), pp.1201-1211.

(9) Tadikonda, S.K. and Baruh, H., Dynamics and Control of a Translating Flexible Beam with a Prismatic Joint, ASME Journal of Dynamic Systems, Measurement, and Control, Vol.114 (1992), pp.422-427.

(10) Yang, K.J., Hong, K.S. and Matsuno, F., Robust Adaptive Boundary Control of an Axially Moving String under a Spatiotemporally Varying Tension, Journal of Sound and Vibration, Vol.273 (2004), pp.1007-1028.

(11) Chung, C.H. and Tan, C.A., Active Vibration Control of the Axially Moving String by Wave Cancellation,
ASME Journal of Vibration and Acoustics, Vol.117 (1995), pp.49-55.

(12) Lee, S.Y. and Mote, C.D., Jr., Vibration Control of an Axially Moving String by Boundary Control, ASME Journal of Dynamic Systems, Measurement, and Control, Vol.118 (1996), pp.66-74.

(13) Fung, R.F., Wu, J.W. and Lu, P.Y., Adaptive Boundary Control of an Axially Moving String System, ASME Journal of Vibration and Acoustics, Vol.124 (2002), pp.435-440.

(14) Li, Y., Aron, D. and Rahn, C.D., Adaptive Vibration Isolation for Axially Moving Strings: Theory and Experiment, Automatica, Vol.38, No.3 (2002), pp.379390.

(15) Choi, J.Y., Hong, K.S. and Yang, K.J., Exponential Stabilization of an Axially Moving Tensioned Strip by Passive Damping and Boundary Control, Journal of Vibration and Control, Vol.10 (2004), pp.661-682.

(16) Hong, K.S., Kim, C.-W. and Hong, K.T., Boundary Control of an Axially Moving Belt System in ThinMetal Production Line, International Journal of Control, Automation, and Systems, Vol.2, No.1 (2004), pp.55-67.

(17) Lee, S.Y. and Mote, C.D., Jr., Wave Characteristics and Vibration Control of Translating Beams by Optimal Boundary Damping, ASME Journal of Dynamic Systems, Measurement, and Control, Vol.121 (1999), pp.18-25.

(18) Li, Y. and Rahn, C.D., Adaptive Vibration Isolation for Axially Moving Beams, IEEE Transactions on Mechatronics, Vol.5, No.4 (2000), pp.419-428.

(19) Fung, R.F., Chou, J.H. and Kuo, Y.L., Optimal Boundary Control of an Axially Moving Material System, ASME Journal of Dynamic Systems, Measurement, and Control, Vol.124 (2002), pp.55-61.

(20) Fard, M.P. and Sagatun, S.I., Exponential Stabilization of a Transversely Vibrating Beam via Boundary Control, Journal of Sound and Vibration, Vol.240, No.4 (2001), pp.613-622.

(21) Wickert, J.A. and Mote, C.D., Jr., On the Energetics of Axially Moving Continua, Journal of the Acoustical Society of America, Vol.85, No.3 (1989), pp.13651368.

(22) Benaroya, H., Mechanical Vibration: Analysis, Uncertainties, and Control, (1998), p.249, Prentice-Hall, Inc., NJ.

(23) Munson, B.R., Young, D.F. and Okiishi, T.H., Fundamentals of Fluid Mechanics, (1998), p.214, Wiley, NY.

(24) Mitrinovic, D.S., Pecaric, J.E. and Fink, A.M., Inequalities Involving Functions and Their Integrals and Derivatives, (1991), p.67, Kluwer Academic, Dordrecht.

(25) Pazy, A., Semigroups of Linear Operators and Applications to Partial Differential Equations, (1983), p.116, 145, Springer-Verlag, NY.

(26) Saperstone, S., Semidynamical Systems in Infinite Dimensional Spaces, (1981), p.78, Springer-Verlag, NY. 